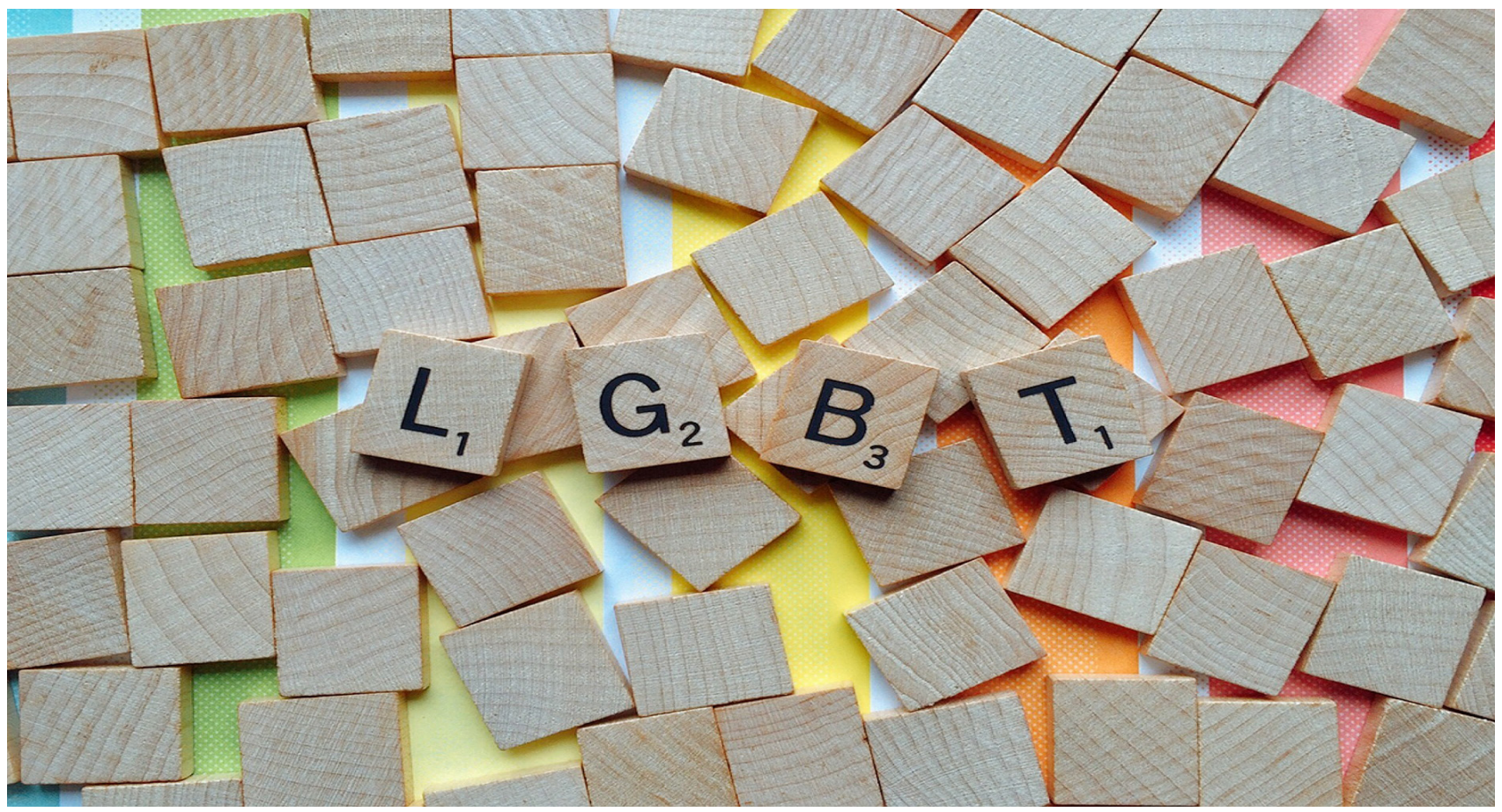

\title{
Bullying homofóbico entre estudiantes de la facultad de derecho de la Fundación Tecnológica Antonio de Arévalo
}

\section{Bullying Homophobic among students of the Faculty of Law of the Technological Foundation Antonio de Arévalo}

María Cristina Arzuza-Buelvas

Magister en Administración de Empresas mcarzuza@yahoo.es, Fundación Tecnológica Antonio de Arévalo- Tecnar, Cartagena de Indias, Colombia

\section{Sandy Stella Sierra-Flórez}

Magister en Educación, Sandy.sierra@gmail.com, Fundación Tecnológica Antonio de Arévalo Cartagena de Indias, Colombia

\section{Rosana Patricia Barvó-Fernández}

Especialista en servicio al cliente, rosana.barvo@gmail.com, Fundación Tecnológica Antonio de Arévalo- Tecnar, Cartagena de Indias, Colombia. 


\section{Resumen}

El bullyng no solo ocurre en escuelas primarias y secundarias, del mismo modo puede ocurrir en las universidades, las personas creen que estos problemas solo ocurren en la infancia y que se supera porque según ellos son cosas de niños". En el siguiente artículo se muestran los resultados obtenidos de la investigación realizada hacia los estudiantes de segundo a décimo semestre de la facultad de Derecho de la Fundación Tecnológica Antonio de Arévalo, sobre los casos de bullying homofóbico que se han presentado y la forma en cómo la Institución respalda a las víctimas y ejerce acciones disciplinarias en contra de los agresores. Para obtener los resultados, se hizo uso de una encuesta, un diseño bibliográfico y se aplicó la observación directa que permitieran identificar cuáles son las causas y los detonadores más frecuentes de dichas situaciones. Por lo tanto, los resultados obtenidos son el reflejo de las opiniones y la percepción que los mismos estudiantes tienen sobre el bullying ejercido hacia personas con una orientación sexual diferente a la propia. Asimismo, queda claro cómo este fenómeno se ha vuelto constante entre estudiantes universitarios y las medidas que toda institución debería implementar.

Palabras clave: Bullying, homofobia, identificación, institución, intimidación, cultura.
Abstract

Bullyng not only occurs in primary and secondary schools, it can also happen in universities, people believe that these problems only occur in childhood and that it is overcome because according to them because "they are children's things". The following article shows the results obtained from the research carried out towards the students from second to tenth semester of the Faculty of Law of the Antonio de Arévalo Technology Foundation, on the cases of homophobic bullying that have been presented and the way in which the Institution supports the victims and exercises disciplinary actions against the aggressors. To obtain the results, a survey, a bibliographic design was used and direct observation was applied to identify the causes and the most frequent triggers of these situations. Therefore, the results obtained reflect the opinions and the perception that the students themselves have about the bullying towards people with a sexual orientation different from their own. It is also clear how this phenomenon has become constant among university students and the measures that every institution should implement.

Keywords: Bullying, homophobia, identification, institution, intimidation, culture. 
Introducción

Los casos de bullying en Colombia suelen ser habituales en las instituciones escolares de secundaria y primaria. Los problemas sociales, económicos, culturales y académicos, en la mayoría de los ocasiones, son los detonadores de dichos comportamientos y es por esto que las diferentes escuelas luchan constantemente para tratar de disminuir el índice de casos de matoneo entre sus estudiantes. Desde hace varios años, distintos investigadores se han dedicado a reconocer, analizar y acabar con el matoneo escolar y aprender a identificar cuáles son las causas que los originan para poder establecer planes de mejoramiento que le den solución a dicha problemática; sin embargo, aunque este problema ha sido expuesto abiertamente en las instituciones de Básica Primaria y Secundaria, las universidades han pasado casi desapercibidas en cuanto al registro de estudiantes que hayan expuesto abiertamente haber sufrido de bullying durante su formación profesional; que puede verse confirmada según [1] en la predilección de estudiantes por cursar electivas de crecimiento humano y cultura en los diferentes programas académicos universitarios. No obstante, esto no quiere decir que no hayan existido o existan, pues investigaciones como éstas han evidenciado lo contrario y la manera en cómo se percibe de forma más matizada o encubierta.

Los jóvenes que inician su proceso de formación profesional se ven expuestos a una serie de conflictos provenientes generalmente, de sus mismos compañeros del aula de clases. En esta etapa del desarrollo social e intelectual del ser humano es en donde los grandes cambios tanto físicos, psicológicos como culturales se arraigan y establecen de formas más o menos fijas en las identidades de cada persona y es allí en donde se empiezan a consolidar los gustos, las actividades, los conocimientos, las habilidades y demás capacidades que se desarrollan hasta llegar a la adultez [2].

Por ser esta una etapa tan conflictiva y decisiva en el devenir social, es la más susceptible a cambios y a la vez, el momento en que se encuentran más expuestos a las influencias del contexto en donde se desenvuelven dichos individuos. Es por estoque los casos de intimidación verbal o física tienen fuertes consecuencias o repercusiones en la percepción de realidad que un estudiante joven puede tener. En las universidades, los casos de matoneo aunque suelen ser camuflados entre los mismos estudiantes, directores y cuerpo administrativo, no significa que sean aceptados y pasen por alto ante los ojos de todos, pues hoy día a través de las redes sociales o demás fuentes de información masiva, suelen conocerse cada vez más nuevos sucesos de intimidación universitaria y pueden conllevar a que los estudiantes puedan abandonar los procesos formativos debido a problemas derivados como consumo de sustancias psicoactivas [3].

Muchas veces, los mecanismos de camuflaje se traslucen entre el juego, la burla social y la distracción 'sana' del otro, al ser métodos de agresión más definidos, pulidos y establecidos que los ya conocidos, puesto que los comentarios, las burlas y las agresiones son más pensados, analizados y dichos para afectar directamente la susceptibilidad de la víctima. Ya no se trata del mismo bullying escolar entre niños y pre- adolescentes sino entre adolescentes y adultos.

En la Fundación Tecnológica Antonio de Arévalo, se realizó una investigación para determinar si se han presentado o no casos de bullying homofóbico entre los estudiantes de segundo a décimo semestre de la facultad de Derecho para poder establecer de qué forma la universidad se encuentra preparada o no para confrontar este tipo de conflictos $y$, si disciplinariamente tienen estatutos que 
beneficien y castiguen a la víctima y a su agresor, respectivamente.

El concepto de homofobia. De acuerdo a [4] las reacciones de los adolescentes homosexuales a la homofobia escolar son de varios tipos. La homofobia es toda actitud, ofensiva, denigrante, racista hacia alguna persona $o$ algo por su conducta u orientación sexual, la homofobia es una fobia: es simplemente un temor morboso e irracional produce un comportamiento de escape o deseo de dañar el estimulo de la fobia o cualquier cosa que se le parezca.

La Homofobia es simplemente: una fobia un temor morboso e irracional que provoca un comportamiento irracional de huida o el deseo de destruir el estímulo de la fobia o cualquier cosa que la recuerde, muchos niños jóvenes y adolescentes hacen una vida doble para vivir en un medio hostil, aceptando aparentemente el grupos social y pasar desapercibido, en cambio oros pueden entrar en un transe de o proceso de desestabilización emocional que puede llevarlos a la autodestrucción.

El bullyng homofóbico. La violencia de género y bullying homofóbico desde el punto de vista de [5] el bullying homofóbico es un tipo específico de violencia sexual que está basado en la orientación sexual y/o identidad de género. La violencia es generalmente producida entre estudiantes, aunque también puede generarse por docentes y personal que trabaja en las escuelas, universidades e instituciones hacia los estudiantes que demuestren algún tipo de conducta, identidad y/u orientación sexual de género diferente al patrón de conducta predominante.

La homofobia en la universidad. El Bullying o acoso escolar en las universidades es un término definido por varios autores: [6], [7] y [8]..."Bullying, es una conducta de persecución física y/o psicológica que realiza un estudiante contra otro, al que escoge como víctima de repetidos ataques de agresión, con insultos prolongados rechazo e intimidación y rechazo social. Muchas veces las personas que acosan a otros, ya sea por su condición sexual, religión, cultura, fijación política, son personas que tienen la necesidad de ser los mejores del grupo o sienten que deben tener el control de la mayoría de sus compañeros o se sienten más fuertes o que tienen ínfulas de superioridad, a esto se suman más estudiantes para ser aceptados o reconocidos por los demás sin anteponer sus sentimientos, sus valores o son manejados o motivados a realizar estos actos de acoso hacia sus compañeros.

En consecuencia se puede determinar que una de las causas de la deserción universitaria, es el bullyng, en la referencia [9] se explica como es importante conocer las bases teóricas que permitan ver desde una perspectiva global las causas que determinan el abandono repentino de los estudiantes en las instituciones. Una de causas que determinan la deserción o abandono de las instituciones es el constante acoso, abuso excesivo verbal y de manoteo, de los estudiantes a otros o puede motivar a la expulsión [10].

\section{Materiales y métodos}

Autores como [11] explican desde un punto de vista científico, la investigación es un proceso que nos permite aplicar el método y las técnicas científicas adecuadas para encontrar respuesta al problema inicialmente planteado. La metodología utilizada en esta investigación es descriptiva basado en un enfoque descriptivo, realizado mediante una encuesta a un grupo de estudiantes de la Universidad de Tecnar de la facultad de derecho dando a conocer situaciones, conflictos y convivencias que ocurren entre los estudiantes, docentes y personal que trabaja dentro de la institución con respecto al bullyng homofóbico. 
Bullying homofóbico entre estudiantes de la facultad de derecho de la Fundación Tecnológica Antonio de Arévalo Tecnar

\section{0}

Resultados y discusión

De los 50 encuestados, el $100 \%$ pudo identificar dentro de sus demás compañeros, quiénes son homosexuales, lesbiana u otro; el $35 \%$ admite haber sufrido de bullying ya sea verbal, física o por medio del cyberbullying, que es otra forma de intimidar a través de las redes sociales; el $65 \%$ ha sido testigo del matoneo hacia homosexuales; sólo el 3\% admitió haber ejercido cualquier forma de intimidación hacia algún compañero homosexual; el $100 \%$ coincide en que la universidad no ha ejercido ningún tipo de acciones hacia las personas que se ven envuelta en los casos de matoneo; el $76 \%$ conoce los reglamentos que establece el código de ética y convivencia de Tecnar4; el $45 \%$ vela por el cumplimiento de los estatutos que Tecnar implementa en la institución; el $87 \%$ considera que deben existir casos severos para los agresores; el 95\% opina que si no se establecen medidas de control, los casos de matoneo sólo irán en aumento; y, solamente el $62 \%$ dio una alternativade solución para anexarla en el código de convivencia y ética de la institución. Todos estos datos se pueden observar en la siguiente tabla:

Tabla 1. Encuesta

\begin{tabular}{|c|c|c|c|}
\hline \multirow{2}{*}{ Preguntas } & \multicolumn{3}{|c|}{ Respuestas } \\
\hline & Sí & No & $\%$ \\
\hline $\begin{array}{l}\text { ¿Tiene compañeros con diferente orientación sexual a la suya? } \\
\text { ¿Ha sido víctima de bullying homofóbico (matoneo por su condición gay }\end{array}$ & 50 & 0 & $100 \%$ \\
\hline $\begin{array}{l}\text { o lesbiana) por parte de sus compañeros de estudio, docentes o } \\
\text { administrativos? }\end{array}$ & 17 & 33 & $35 \%$ \\
\hline $\begin{array}{l}\text { ¿Ha sido testigo de casos de bullying hacia los homosexuales que estudian } \\
\text { en la facultad de Derecho? }\end{array}$ & 32 & 18 & $65 \%$ \\
\hline $\begin{array}{l}\text { ¿Ha agredido verbal, física o emocionalmente a alguno de sus compañeros } \\
\text { por tener una orientación sexual diferente a la suya? }\end{array}$ & 2 & 48 & $4 \%$ \\
\hline $\begin{array}{l}\text { ¿La Institución Tecnológica Antonio de Arévalo ha impuesto castigos o ha } \\
\text { realizado alguna acción en contra de los casos de bullying que se } \\
\text { presentan en sus instalaciones? }\end{array}$ & 50 & 0 & $100 \%$ \\
\hline $\begin{array}{l}\text { ¿Conoce los reglamentos que Tecnar imparte en el Código de ética y } \\
\text { convivencia? }\end{array}$ & 38 & 12 & $76 \%$ \\
\hline $\begin{array}{l}\text { ¿Cómo estudiante de Derecho, vela por el cumplimiento de todos los } \\
\text { estatus que Tecnar establece? }\end{array}$ & 22 & 28 & $45 \%$ \\
\hline $\begin{array}{l}\text { ¿Considera que deben existir castigos severos para las personas que } \\
\text { agreden e intimidan a otros por su condición sexual? }\end{array}$ & 43 & 7 & $87 \%$ \\
\hline $\begin{array}{l}\text { ¿Considera que los casos de bullying homofóbico irán en aumento si no se } \\
\text { aplican normas preventivas o cualquier otra actividad que lo controle? }\end{array}$ & 47 & 3 & $95 \%$ \\
\hline $\begin{array}{l}\text { ¿Qué norma propondría usted para anexarla dentro del Código de ética y } \\
\text { convivencia que Tecnar establece? }\end{array}$ & 31 & 19 & $62 \%$ \\
\hline
\end{tabular}

Como afirma [12] "La homofobia encierra a las personas en roles de género rígidos y estáticos que disminuyen la creatividad y la capacidad de expresión". Queda claro que, dentro la institución los casos de bullying hacia personas homosexuales sí han ocurrido pero para la gran mayoría han pasado desapercibidas dichas agresiones, puesto que, durante la observación 
directa que se realizó, estas no son tanto de índole física sino verbal por medio de la burla y los comentarios malintencionados que se escuchan al referirse a dichas personas. Los comentarios, aunque para la gran mayoría son una forma de juego e interacción 'normal' y común entre ellos, éstos afectan la autoestima y susceptibilidad de la persona referida.

En ocasiones, se pudo observar que las mismas víctimas hacen ver que no lo son y no les afectan los comentarios homofóbicos que escuchan y siguen siendo parte del juego con el fin de no sentirse más excluidos de lo que están.

Desde el punto de vista, [13] señala que los seres humanos son por naturaleza malos, crueles y violentos: "el hombre nace salvaje y podría ser un peligro universal durante toda su vida si no lo meten a un aula de clases para domesticarlo... el hombre es malo por naturaleza, y si la sociedad no lo educa y no lo corrige, entonces comienza a padecer la intolerancia, la maña y la crueldad de los salvajes". Ahora bien, uno de los métodos de matoneo directo y público que repercute en grandes masas y de forma más severa es a través del ciberacoso o cyberbullying en donde la creación de memes, que son imágenes caricaturescas y animadas que pueden convertirse en ofensivas, ha sido la plataforma para generar humillación y expresar los pensamientos homofóbicos que la gran mayoría e los estudiantes sienten hacia sus compañeros. Estas imágenes al ser expuestas en redes sociales como el Facebook, whatsapp, Space, Messenger, instagram, twitter, entre otras, son vistas por todos los grupos sociales en que tanto las victimas como agresores se desenvuelven.

Cuando se hicieron preguntas aleatorias a estudiantes conocidos sobre lo que pensaban si en la universidad se daban o conocen casos de bullying homofóbico muchos dijeron que no tenían conocimiento de éstos y que conocían a compañeros homosexuales pero que todos los comentarios que se hacían eran bajo la línea del respeto. Ahora, lo que se considera "línea del respeto" para muchos, para otros no lo es, sobre todo para los directamente involucrados. También, el hecho de que existe la necesidad de crear esos comentarios, memes o chistes que atentan contra la integridad del ser homosexual se evidencia un problema latente pues, la sola idea de generarlos es agresión, ofensa y discriminación.

La universidad, por su parte, no cuenta con ningún estatuto en su Código de ética y convivencia que se encuentre relacionado con acciones disciplinarias contra los agresores o alguna que beneficie a los agredidos. Los únicos apartes que se pueden tener en cuenta están referidos al mal comportamiento, a malas acciones, al incumplimiento de las reglas, etc. Pero ninguna referida al bullying como tal. A continuación se exponen algunas de las normas establecidas en el código de ética y convivencia de Tecnar a las cuales los estudiantes se deben atener tal como se menciona en el primer y segundo punto:

\section{Primer punto}

- Sembrar valores institucionales con enfocados en la honestidad, humildad, respeto y la armonía sin ningún tipo de discriminación como bases fundamentales para el desarrollo de la convivencia de la vida universitaria.

\section{Segundo punto}

- Evitar conductas inapropiadas, obscenas o indecorosas que apoyen la provocación para que se causen actitudes o situaciones conflictivas de caos en las instalaciones de la Fundación o sus inmediaciones.

- Evitar el uso de expresiones inadecuadas o denigratorias, agresión verbal, uso de insultos, 
Bullying homofóbico entre estudiantes de la facultad de derecho de la Fundación Tecnológica Antonio de Arévalo Tecnar

\section{2}

intimidación, hacia cualquier miembro de la comunidad tecnarista o la propia Institución.

En este mismo documento también existen disposiciones referidas a los docentes como:

- No admitir el acoso, hostigamiento con miras a avances sexuales, de favores sexuales y conductas verbales o físicas que constituyan una persecución sexual, bajo amenaza de castigo físico o académico y bajo promesa de premios académicos, intentos de seducción basados en el ejercicio del poder que otorga una posición en la Institución.

Asimismo, como estos enunciados que están dirigidos al cuerpo administrativo:

- Propiciar la fraternidad en la comunidad Tecnarista, evitandoasí comentarios yactitudes que deterioren las relaciones interpersonales y el trabajo de equipo en la Institución.

- Difundir la honestidad y actuar con objetividad y justicia en los juicios valorativos éticos, morales y legales que afecten la sana convivencia entre los miembros de la comunidad Tecnarista.

Continuamente hay otras pautas referidas a los castigos severos que reciben en caso del incumplimiento del mismo. De igual forma, dentro de los principios y valores se mencionan la tolerancia y el respeto pero como queda claro no existe ninguno referido directamente a los casos de bullying encontrados en la institución.

Todo ente o institución debe tener reglamentos que beneficien a sus estudiantes, el no tenerlos hace que se ejerzan mecanismos fuera de lo normal y que no se tenga un control o libertad plena de su condición de estudiante y persona que se instruye para formarse profesionalmente, es así que dentro de los resultados obtenidos se puede percatar que la percepción que tienen los estudiantes, que tal veznoseencuentraninvolucrados directamente con los casos de matoneo, consideran que si no se implementan fuertes normas en contra de los agresores, los casos de bullying sólo aumentaran considerablemente, pues, ya otras universidades lo han experimentado.

Varias publicaciones de periódicos de reconocimiento en el país han expuesto diversos artículos para referirse a estos fenómenos y situaciones ocurridas a estudiantes de bajos recursos y de orientación sexual diferente. Según [14] afirma que "La homofobia es la causa principal de matoneo en el contexto académico, hecho que resulta más complejo cuando los docentes no muestran autoridad en las aulas para que se respeten las condiciones propias de cada ser." Además expone el caso de estudiantes que hablaron sobre sus experiencias del bullying homofóbico que recibieron:

Hijos de padres del mismo sexo, jóvenes con problemas de obesidad o anoréxicos, por su formadevestir, porsucreenciareligiosa... Casos como estos son comunes en las universidades y Tecnar, aunque hasta la fecha no se ha visto envuelto en ninguno de gran magnitud si no comienza a implementar medidas en contra del cyberbullying pasará a formar parte de este amplio grupo.

\section{Conclusiones}

El papel que asume la universidad dentro del bullying es fundamental pues, es el responsable de las respuestas obtenidas por los agresores y las víctimas en una situación conflictiva. Además, una de los principios que rigen a Tecnar es el de anteponer ante todo el bienestar de sus estudiantes y velar por la buena convivencia entre todos los involucrados en su formación. Es casi el deber de los docentes en ser accesibles y convertirse en puentes o intermediarios para que los estudiantes puedan ser más abiertos con respecto a los problemas que tengan 
dentro o fuera de la institución por medio de la implementación de espacios amenos e igualitarios en donde la participación de todos sea reconocida y valorada.

Se deja claro que una de las principales acciones que debe tomar Tecnar, es iniciar con acciones disciplinarias severas hacia los estudiantes que por más mínima que sea su agresión pero que afecte directamente la integridad del otro, sea homosexual o no, debe recibir un castigo.

De igual forma, esas acciones deben quedar registradas detalladamente en el Código de ética y convivencia para que los viejos y antiguos estudiantes del plantel conozcan.

Los estudiantes de Derecho, al estar relacionados directamente con las leyes y los reglamentos que el ciudadano colombiano debeacatary ejercer, debe estar comprometido al beneficio del otro y que este otro pueda desenvolverse plenamente y con total libertad, pues, se supone que ellos serán los futuros ejercedores de la ley. El hecho que entre ellos se presenten casos de bullying homofóbico en grande o pequeña escala no los hace más o menos vulnerables sino que les abre camino para empezar a implementar normas que controlen, prevengan o erradiquen dichas situaciones problemáticas.

\section{Referencias}

[1] D.A. Rivera y M. Ruiz, "Satisfacción del Estudiantes que Actualmente Cursa Electivas en Bienestar Institucional FESC", Mundo FESC, vol. 5, no. 9, pp. 79-94, 2015

[2] L. Montañez Carrillo y L. Gutiérrez, "Medición de la Madurez de la Gestión del Conocimiento en la Escuela de Ciencias Básicas Tecnología e Ingeniería de la UNAD”, Publicaciones E Investigación, vol. 10, pp. 177-191, 2016

[3] M. Vergel Ortega, J.J. Martínez-Lozano, y E. Ibargüen-Mondragón, "Modelos estimados de análisis de supervivencia para el tiempo de permanencia de los estudiantes de la Universidad Francisco de Paula Santander", Respuestas, vol. 21, no. 2, pp. 24 - 36, jul. 2016

[4] T. Louis-Georges. Diccionario Akal de la Homofobia. Paris: Presses universitaires de France, 2003.

[5] Unesco, "Violencia de género y bullying homofóbico", OVE, Otras Voces en Educación. 2016. [En línea]. Disponible en: http:// otrasvoceseneducacion.org/archivos/122056 [Accedido: 27-jul-2016]

[6] D. Olweus. Conducta de Acoso y Amenaza entre Escolares. Madrid: Morata, 1998

[7] P.K. Smith y S. Sharp. School Bullying: Insights and Perspectives. New York: Routledge, 1994

[8] R. Ortega-Ruiz, "Violencia interpersonal en los centros educativos de enseñanza secundaria. Un estudio sobre el maltrato e intimidación entre compañeros", Revista de Educación, no. 304, pp. 253-280, 1994

[9] A. Gutiérrez-Ortiz, M. García, E.J. BarrientosMonsalve y J.S. Jején, "Factores determinantes en la deserción estudiantil Fesc en el programa de diseño y Modas", Convicciones, vol. 5, no. 10 , pp. 48-54, 2018

[10]A.N. Suárez-Montes, L.E. Fajardo-Cantor, E.J. Barrientos-Monsalve y Y.C. Ríos-Epalza "Estrategias pedagógicas de micro finanzas a los estudiantes del Colegio Municipal Aeropuerto", Convicciones, vol. 08, pp.17-21, Julio - Diciembre 2017. [En línea]. Disponible en:https://www.fesc.edu.co/Revistas/OJS/ index.php/convicciones/article/view/232 


\section{4}

[11] C. Lafuente-Ibáñez y M. EgoscozábalAinhoa "Metodologías de la investigación en las ciencias sociales: Fases, fuentes y selección de técnicas", Revista Escuela de Administración de Negocios, no. 64, pp. 5-18, Septiembre-Diciembre, 2008. [En línea]. Disponible en: https://www.redalyc.org/ articulo.oa? $\mathrm{id}=20612981002$

[12] A.B. Gómez, "Adolescentes gays y lesbianas en riesgo: Aspectos psicosociales. Orientaciones". Revista de Homosexualidades. Adolescencias, no. 8, 2trim, 2004

[13] M.A. Valencia "Sobre el matoneo en la escuela”, El Espectador, [en línea]. Disponible en: https://blogs.elespectador.com/actualidad/ la-casa-encendida/sobre-el-matoneo-en-laescuela [Accedido: 27-jun-2016]

[14] El País, "Bullying, un problema que también afecta a las universidades", 2015. [En línea]. Disponible en: http://www.elpais.com.co/elpais/ cali/noticias/bullying-problema-tambienafecta-universidades. . [Accedido: 27-jun-2016] 